\title{
STRESSES IN COATING WITH GRADIENT INTERLAYER CAUSED BY CONTACT LOADING
}

\author{
Roman KULCHYTSKY-ZHYHAILO, Adam Stanisław BAJKOWSKI ${ }^{*}$
}

*Faculty of Mechanical Engineering, Department of Mechanics and Applied Computer Science, Bialystok University of Technology ul. Wiejska Street 45C, 15-351 Bialystok, Poland

\author{
r.kulczycki@pb.edu.pl, a.bajkowski@pb.edu.pl
}

\begin{abstract}
The three-dimensional problem of elasticity concerning inhomogeneous half-space under normal and tangential loading applied in circular region was considered. The half-space is composed of the homogeneous body and double-layer coating which includes a homogeneous top coat and a gradient interlayer. The solution method is based on the two-dimensional integral Fourier transform. The influence of mechanical properties of coatings component and coefficient of friction on the first principal stress distribution was considered.
\end{abstract}

Key words: Gradient Coating, Three-Dimensional Problem Of Elasticity, Normal Loading, Shear Loading

\section{INTRODUCTION}

To improve the tribological properties of the surface of frictionally cooperating elements the coatings are used. Coatings enable to reduce coefficient of friction and increase resistance to adverse chemical and thermal influence of cooperating elements. Usually Young's modulus of the coating is greater than the modulus of the base. The stress analysis is required for appropriate selection of the coating. The maximum tensile stress is often regarded as the factor which initiates the cracking. In homogeneous coating it could occurs at the interface between coating and base Schwarzer et al. (1999); Bragallini et al. (2003); KulchytskyZhyhailo and Rogowski (2010). To decrease these stresses we can insert the interlayer between top coat and base. The interlayer has Young's modulus between base and top coating moduli. Solution for homogeneous half-space with inhomogeneous coating which properties (Young's modulus) were changing according to power or exponential law is shown in papers: Giannakopoulos and Suresh (1997); Giannakopoulos and Pallot (2000); Aizikovich et al. (2002); Fischer-Cripps (2003); Guler and Erdogan (2004, 2006, 2007); Ke and Wang (2006, 2007); Liu and Wang (2008, 2009); Liu et al. (2008); Choi (2009); Kulchytsky-Zhyhailo and Rogowski (2009). Research describing influence of homogeneous interlayer was carried out by Diao et al. (1996); Lijesh and Amirthagadeswaran (2010). These articles concern onedimensional, two-dimensional or axis-symmetrical problems. Kashtalyan and Menshykova (2009), Kashtalyan et al. (2009) analysed the stresses in three-dimensional problem of loading plate with coating which includes gradient interlayer using the Finite Element Method.

The aim of this work is to carry out the first principal stress $\sigma_{1}$ analysis in three dimensional problem of elasticity concerning normal and tangential loading applied in surface of inhomogeneous half-space. Considered half-space is composed of homogeneous isotropic linear-elastic half-space and double-layer which contains homogeneous top coat and gradient interlayer (Fig. 1). The mechanical properties of the base and top coat were described respectively by Young's moduli $E_{0}$ and $E_{2}$, and constant Poisson's ratios $v_{0}$ and $v_{2}$. Interlayer's mechanical properties are described by Poisson's ratio $v_{1}(z)$ and Young's modulus $E_{1}(z)$, which are changing with distance to surface according to determined dependence.

Between components of considered half-space the ideal contact condition was satisfied. The surface is under normal $p$ and tangential ( $t=f p, f-$ coefficient of friction) loading applied in circular area $\Omega$, described by radius $a$. We assume that the loading distribution is elliptical:

$$
p(x, y)=p_{\max } \sqrt{1-x^{2}-y^{2}}
$$

where: $x, y, z$-dimensionless Cartesian coordinates related to radius of loading area.

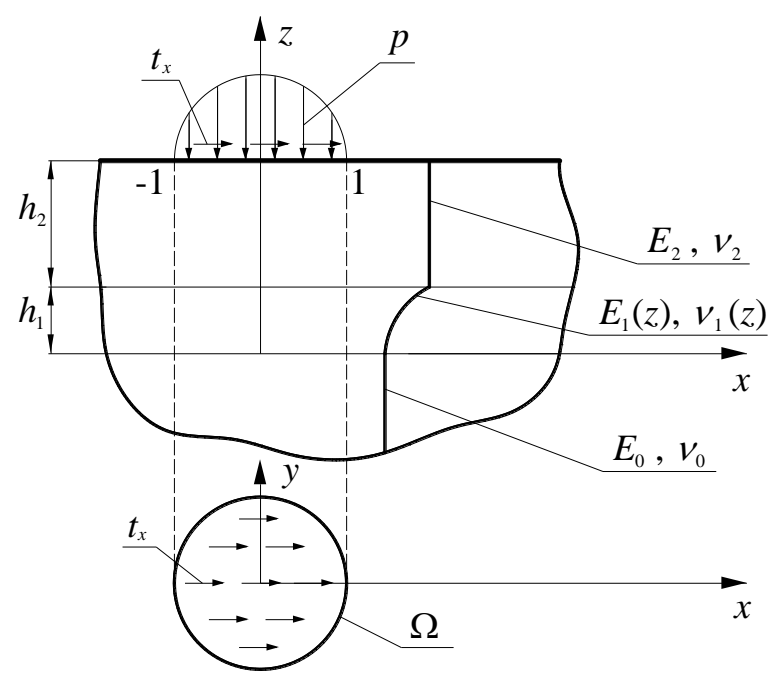

Fig. 1. Scheme of the problem

\section{MATHEMATICAL FORMULATION AND METHOD OF SOLUTION}

The equations of theory of elasticity in interlayer are differential partial equations with various coefficients which analytic solu- 
tions are known only in special cases. Because of it, the gradient coating was replaced by package of homogeneous layers. Young's modulus and Poisson's ratio of each layer are specified through their average in every layer by its thickness. In Kulchytskyy and Bajkowski $(2011,2012)$ papers this approach was presented for cases of one-layer gradient coating which Young's modulus is described respectively by the exponential and power functions. Results obtained using package of the layer were compared with analytical results. Good agreement between results were obtained.

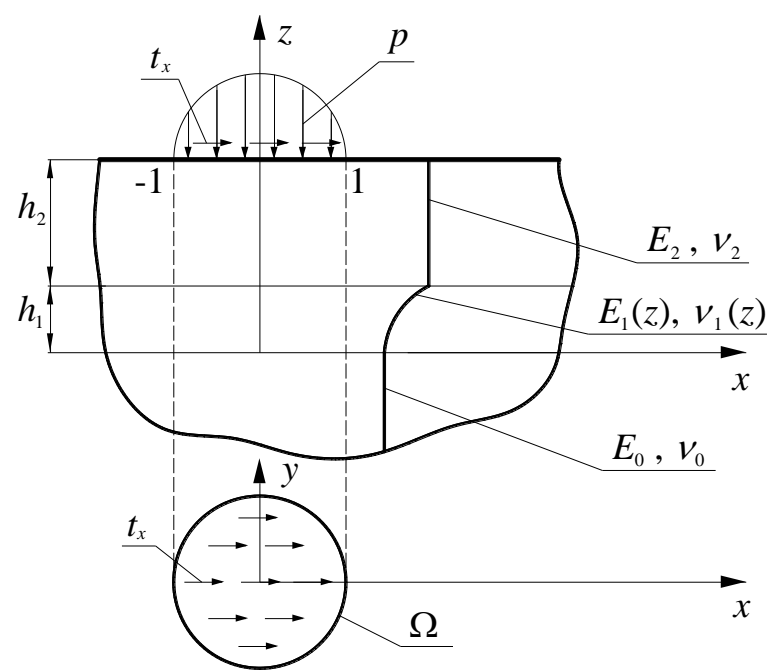

Fig. 2. Modelling of the inhomogeneous coating by package of homogeneous layers

Usage of the Kulchytskyy and Bajkowski $(2011,2012)$ approach to the problem based on solving the elasticity equations in every component of analysing inhomogeneous half-space (Fig. 2).

$\left(1-2 v_{i}\right) \Delta \mathbf{u}^{(i)}+\operatorname{grad} \operatorname{div} \mathbf{u}^{(i)}=0$

Solution of the equations (2) should satisfy the boundary conditions:

- loading the surface of the considered half-space

$\sigma_{x z}^{(n)}(x, y, z=h)=t_{x}(x, y) H(x, y)$

$\sigma_{y z}^{(n)}(x, y, z=h)=0$

$\sigma_{z z}^{(n)}(x, y, z=h)=-p(x, y) H(x, y)$

- perfect contact condition between components of half-space

$\mathbf{u}^{(i+1)}\left(x, y, z=z_{i}\right)=\mathbf{u}^{(i)}\left(x, y, z=z_{i}\right), i=0, \ldots, n-1$

$\boldsymbol{\sigma}^{(i+1)}\left(x, y, z=z_{i}\right) \cdot \mathbf{n}=\boldsymbol{\sigma}^{(i)}\left(x, y, z=z_{i}\right) \cdot \mathbf{n}, i=0, \ldots, n-1(7)$

- decline the value of the components of displacements in infinity

$\mathbf{u}^{(i)}(x, y, z) \rightarrow 0, x^{2}+y^{2}+z^{2} \rightarrow \infty, i=0,1, \ldots, n$.

In equations (2)-(8) we introduce symbols $\Delta=\partial^{2} / \partial x^{2}+\partial^{2} / \partial y^{2}$, $\mathbf{u}^{(i)}$ - dimensionless vector of displacement related to $a$ parameter; $\sigma^{(i)}$ - stress tensor; indexes $i=0,1, \ldots, n$ characterize parameters and functions respectively in base, in the layers of the package $i=1,2, \ldots, n-1$ and in the top layer $i=n$; $\mathbf{n}=(0,0,1) ; H(x, y)-$ Heaviside step function $(H(x, y)=1$, when $(x, y) \in \Omega$ and $H(x, y)=0$, when $(x, y) \notin \Omega) ; h-z$ coordinate of the surface of the inhomogeneous half-space ( relation of the coating thickness to the radius of loading area), $z_{0}=0, z_{i}-z$ coordinate of upper surface of $i$ layer of the package.

The solution of the boundary problem was constructed using the two-dimensional integral Fourier transform, similar to the described in the Kulchytskyy and Bajkowski $(2011,2012)$ works. General solution in a transform space contains $6 n+3$ unknown functions of integral transformation parameters. These functions were obtained computing the results of the system of the linear equations formed through fulfilment of the boundary conditions (3)-(7) in a transform space. Using the inverse integral Fourier transform the relationships between described components of stress tensor were obtained in two-dimensional integrals form. These integrals were calculated introducing polar coordinate in the space of integral transform parameters. Integrals along angular coordinate were calculated analytically. Integrals along radial coordinate in internal points of considered inhomogeneous half-space were calculated numerically using the Gaussian quadrature. During calculating the integrals which described stresses in the surface $(z=h)$ we took into consideration the asymptotic behaviour of the integrand while the radial coordinate was approaching infinity.

\section{RESULTS ANALYSIS}

The results depend on dimensionless parameters: coefficient of friction $f$, thickness of coating $h$ to thickness of interlayer $h_{1}$ ratio, Young's modulus ratio $E_{2} / E_{0}$, Poisson's ratio $v_{0}$ and $v_{2}$, and functions described change of Young's modulus and Poisson's ratio in interlayer. To reduce number of parameters we assume that Poisson's ratio in the whole considered half-space is constant and equals $1 / 3$, therefore $v_{0}=v_{2}=1 / 3, v_{1}(z) \equiv 1 / 3$. Moreover we assume relative high difference between base and top coat Young's modulus $E_{2} / E_{0}=4$

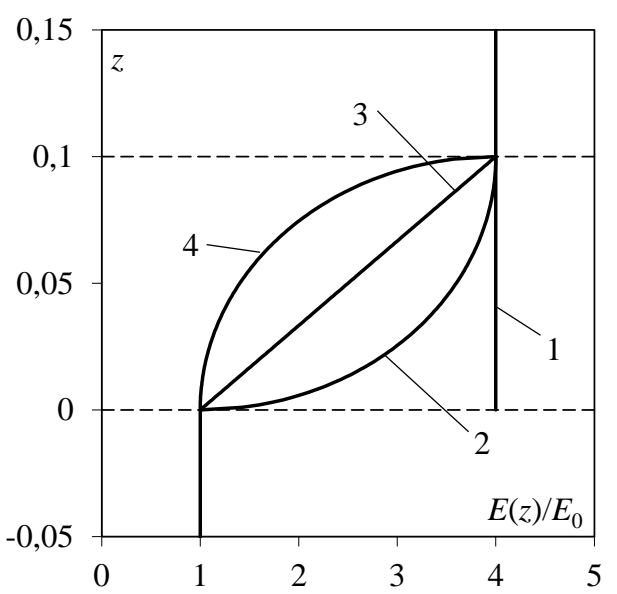

Fig. 3. Diagram of the functions described change of Young's modulus in considered coating. 1 - coating without interlayer; 2-4 - Young's modulus described respectively by the equations (9)-(11) 

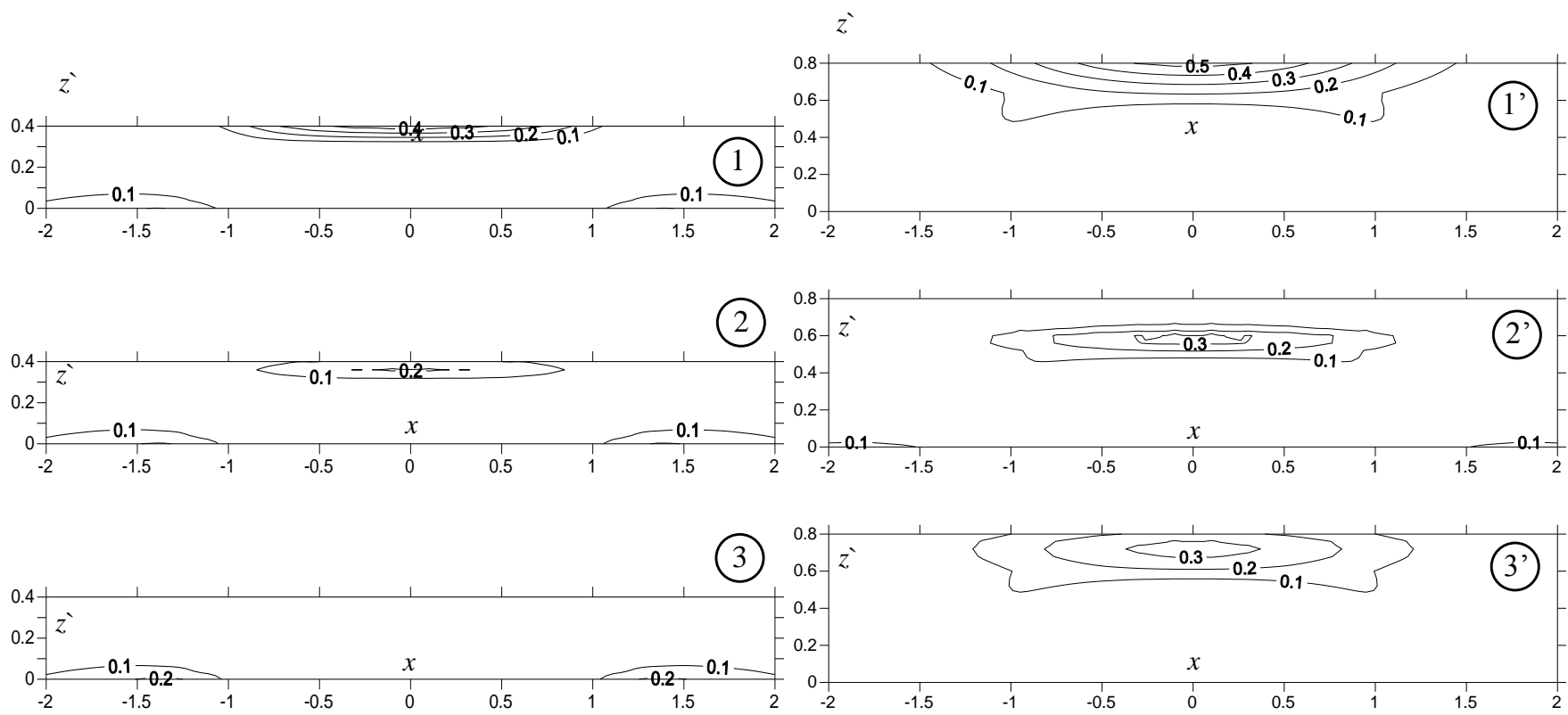

(4)
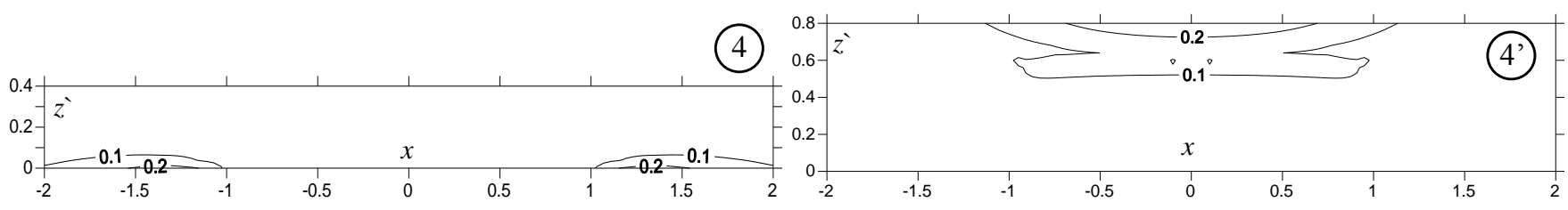

Fig. 4. Distribution of first principal stress $\sigma_{1} / p_{\max }$ in the $x z$ plane, $f=0.1-4$ - like in Fig. 3.,

Fig. 4.1-4.4: $h=0.4$; Fig. 4.1'-4.4': $h=0.8\left(z^{\prime}=h-z\right)$.

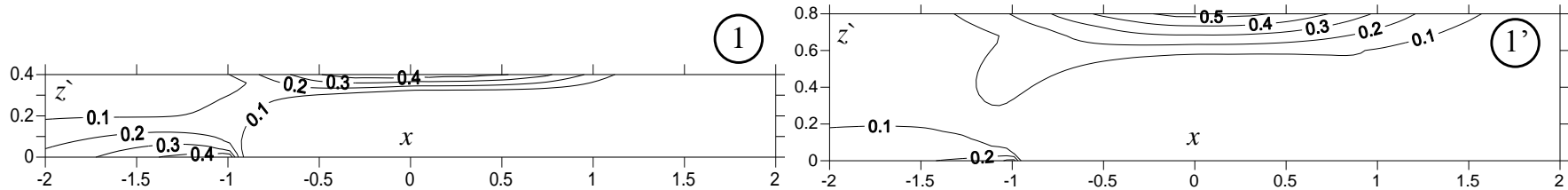

(2)
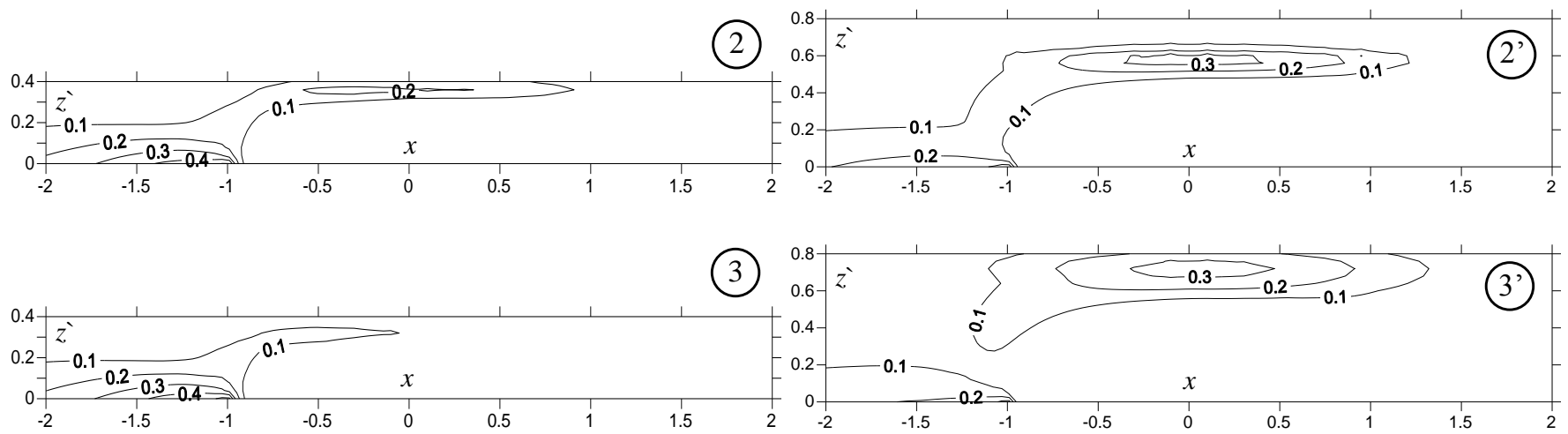

(4)

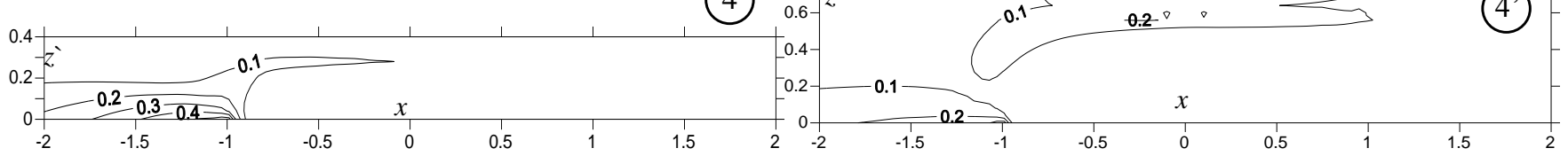

Fig. 5. Distribution of first principal stress $\sigma_{1} / p_{\max }$ in the $x z$ plane, $f=0,25.1-4-$ like in Fig. 3.,

Fig. 5.1-5.4: $h=0.4$; Fig.5.1'-5.4': $h=0,8\left(z^{\prime}=h-z\right)$.

For analysis we take following values: coefficient of friction $f=$ 0 and $f=0,25$; Thickness of components of the coating $h_{1} / h=$ $1 / 4, h=h_{1}+h_{2}=0.4$ or 0.8 . We consider the coatings (Fig. 3 ): without interlayer $\left(E_{1}=E_{2}\right)$ and when its Young's modulus is described by the relationships: 


$$
\begin{aligned}
& E_{1}(z)=E_{0}+\left(\frac{2 z}{h}-\left(\frac{z}{h}\right)^{2}\right)^{1 / 2}\left(E_{2}-E_{0}\right) \\
& E_{1}(z)=E_{0}+\frac{z}{h}\left(E_{2}-E_{0}\right) \\
& E_{1}(z)=E_{2}-\left(1-\left(\frac{z}{h}\right)^{2}\right)^{1 / 2}\left(E_{2}-E_{0}\right)
\end{aligned}
$$

Dependences (9)-(11) were chosen so that Young's modulus at the interface between components does not discretely change.

During analysis we have focused at the distribution of first principal stress $\sigma_{1}$ in coating in $x z$ plane (Fig. 4 and Fig. 5), including at the surface on the line $y=0, z=h$ (Fig. 6). In the Fig. 4 and Fig. 5 the tensile stress with values $\sigma_{1} / p_{\max }>0,1$ was shown.
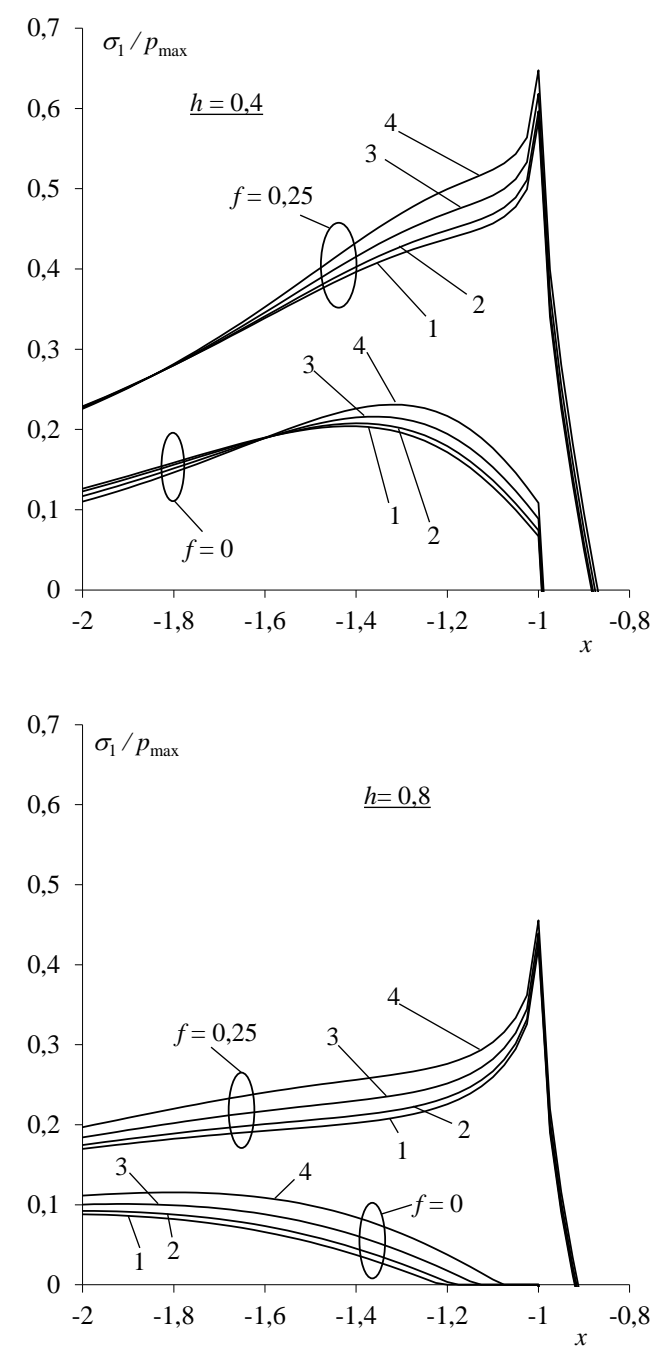

Fig. 6. $\sigma_{1} / p_{\max }$ stress in the external surface of the considered half-space. Lines 1-4 - like in Fig. 3.

\section{SUMMARY}

In the considered coatings, like in homogeneous coatings, tensile stresses may mostly occur in two areas (Fig. 4 and Fig. 5):
1) in the unbiased surface of considered inhomogeneous halfspace, 2) in the surroundings of boundary between coatings and base. At some mechanical properties the area of the tensile stresses was displaced from interface to internal zone of the coating (Fig. 4.2'). The tangential loading causes appreciable increase of level of the tensile stresses in the surface $z=h$ (Fig. $6)$, the area of tensile stress in surface occurs on the left side $(x<0)$ (Fig. 5). In surroundings of boundary between coatings and base the influence of friction is small (Fig. 4 and Fig. 5).

Occurrence of the interlayer and its properties have significant influence on tensile stress distribution in considered medium. The highest tensile stress at the interface between a coating and a base occurs in cases without the interlayer (Fig. 4.1 and Fig. 5.1). The highest reduction of this stress is when interlayers Young's modulus is described by the (10) and (11) equations (Fig. 4.3; 4.4; 5.3 and 5.4).

For all of analysing coatings the tensile stress distribution in the external surface was similar. For coatings of greater thickness stresses in the external surface have smaller values. Occurrence of the gradient interlayer causes insignificant increase of tensile stress in this surface (Fig. 6). Highest increase is when Young's modulus of interlayer is described by the equation (11).

\section{REFERENCES}

1. Aizikovich S.M., Alexandrov V.M., Kalker J.J., Krenev L.I., Trubchik I.S. (2002) Analytical solution of the spherical indentation problem for a half-space with gradients with the depth elastic properties, Int. J. Solids Struct., 39, 2745-2772.

2. Bragallini G.M., Cavatorta M.P., Sainsot P. (2003) Coated contact: a strain approach, Tribology Int., 36, 935-941.

3. Choi H. J. (2009) On the plane contact problem of a functionally graded elastic layer loaded by a frictional sliding flat punch, Mechanics Research Communications, Arch Appl Mech, 78, 267282.

4. Diao D.F., Sawaki Y., Suzuki H. (1996) Effect of interlayer on maximum contact stresses of hard coating under sliding contact, Surface and Coatings Technology, 86-87, 480-485.

5. Fischer-Cripps A.C. (2003) Analysis of instrumented indentation test data for functionally graded materials, Surface \& Coatings Technology, 168, 136-141.

6. Giannakopoulos A.E., Pallot P. (2000) Two-dimensional contact analysis of elastic graded materials, J. Mech. Phys. Solids, 48, 15971631.

7. Giannakopoulos A.E., Suresh S. (1997) Indentation of solids with gradients in elastic properties: Part II. Axisymetric indenters, Int. J. Solids and Structures, 34, 2392-428.

8. Guler M.A., Erdogan F. (2004) Contact mechanics of graded coatings, Int. J. Solids Struct., 41, 3865-3889.

9. Guler M.A., Erdogan F. (2006) Contact mechanics of two deformable elastic solids with graded coatings, Mechanics of Materials, 38, 633-647.

10. Guler M.A., Erdogan F. (2007) The frictional sliding contact problems of rigid parabolic and cylindrical stamps on graded coatings, Int. J. Mech. Sci., 49, 161-182.

11. Kashtalyan M., Menshykova M. (2009) Effect of a functionally graded interlayer on three-dimensional elastic deformation of coated plates subjected to transverse loading, Composite Structures, 89, 167-176.

12. Kashtalyan M., Menshykova M., Guz I.A. (2009) Use of a Functionally Graded Interlayer to Improve Bonding in Coated Plates, Journal of Adhesion Science and Technology, 23, 15911601. 
13. Ke L.L., Wang Y.S. (2006) Two-dimensional contact mechanics of functionally graded materials with arbitrary spatial variations of material properties, Int. J. Solids Struct., 43, 5779-5798.

14. Ke L.L., Wang Y.S. (2007) Two-dimensional sliding frictional contact of functionally graded materials, Eur. J. of Mech. A/Solids, 26, 171 . 188.

15. Kulchytsky-Zhyhailo R., Bajkowski A. (2011) Elastic half space with inhomogeneous coating under the action of tangential forces, Materials Science, 46(6), 735-746.

16. Kulchytsky-Zhyhailo R., Bajkowski A. (2012) Analytical and numerical methods of solution of three-dimensional problem of elasticity for functionally graded coated half-space, Int. J. Mech. Sci., 54, 105-112.

17. Kulchytsky-Zhyhailo R., Rogowski G. (2009) Axially symmetric contact problem of pressing of an absolutely rigid ball into an elastic half space with inhomogeneous coating, Materials Science, 45(6), 845-858.

18. Kulchytsky-Zhyhailo R., Rogowski G. (2010) Stresses in hard coating due to a rigid spherical indenter on a layered elastic halfspace, Tribology International, 43(9), 1592-1601.

19. Lijesh K.P., Amirthagadeswaran K.S. (2010) Optimizing interlayer and coated film thickness for minimum stress distribution under elastohydrodynamic lubrication condition, Proceedings of the
International Conference on Frontiers in Automobile and Mechanical Engineering, 328-334.

20. Liu T.J., Wang Y.S. (2008) Axisymmetric frictionless contact problem of a functionally graded coating with exponentially varying modulus, Acta Mechanica, 199, 151-165.

21. Liu T.J., Wang Y.S. (2009) Reissner-Sagoci problem for functionally graded materials with arbitrary spatial variation of material properties, Mechanics Research Communications, 36, 322-329.

22. Liu T.J., Wang Y.S., Zhang C. (2008) Axisymmetric frictionless contact of functionally graded materials, Mechanics Research Communications, Arch Appl Mech, 78, 267-282.

23. Schwarzer N., Richter F., Hecht G. (1999) The elastic field in a coated half-space under Hertzian pressure distribution, Surf. Coat. Technol., 114, 292-304.

Acknowledgement: This work was carried out within the project „Analysis of stress caused by contact pressure in a homogeneous body with a gradient coating". The Project is financed by the resources of National Science Centre (Poland) given according to decision No. DEC2011/03/N/ST8/04212. 